Running Head: NEUROPSYCHOLOGICAL DISPARITIES IN HIV

Evidence for Neuropsychological Health Disparities in Black Americans with HIV Disease

Jennifer L. Thompson, Ilex Beltran-Najera, Briana Johnson, Yenifer Morales, and Steven Paul Woods*

University of Houston, Houston, Texas

*Corresponding author: Steven Paul Woods, Psy.D. Email: spwoods@uh.edu. Address: 126 Heyne Building, Suite 239D, Houston, TX 77004-5022. Phone: 713-743-6415. 


\begin{abstract}
Objective: Black Americans are at high risk for HIV disease and associated morbidity. However, we know little about the neuropsychological impact and correlates of HIV disease among Black Americans. Methods: Participants included 40 Black persons with HIV (PWH), 83 White PWH, 28 Black HIV- and 64 White HIV- individuals. Neurocognition was measured with raw, sample-based zscores from a clinical battery. Everyday functioning was assessed using self- and clinician-rated measures of cognitive symptoms and activities of daily living. HIV-associated neurocognitive disorders were classified using the Frascati criteria. Results: We observed a significant three-way interaction between HIV, race, and domain on neurocognitive z-scores. This omnibus effect was driven by large effect size decrements in semantic memory and processing speed in Black PWH compared to the other groups. Black PWH participants also demonstrated higher frequencies of HIV-associated neurocognitive disorders as compared to White PWH. Unexpectedly, global neurocognition was negatively related to everyday functioning for White PWH, but not for Black PWH. Conclusions: Systemic disadvantages for Black Americans may combine with HIV disease to compound some neurocognitive impairments in this vulnerable population. Prospective studies are needed to identify better ways to prevent and manage HIVassociated neurocognitive disorders among Black Americans.
\end{abstract}

Keywords: Race; Assessment; Activities of daily living; Health disparities; Cultural psychology Word count: Abstract 196; Manuscript 4,878 


\section{Evidence for Neuropsychological Health Disparities in Black Americans with HIV Disease}

In the United States (U.S.), there is a stark overrepresentation of Black Americans in the HIV epidemic. Approximately 13\% of the U.S. population identifies as Black, but Black Americans comprised $42 \%$ of all new HIV infections and $41 \%$ of all HIV cases in 2018 (CDC, 2020). Several sociocultural factors may combine to increase HIV transmission and infection rates for Black Americans, such as cultural stigmas and higher rates of incarceration (Blankenship et al., 2005, Williams et al., 2009). Medical mistrust stemming from historical atrocities (e.g., the Tuskegee Syphilis study) and lived experiences of racism in the medical system may decrease engagement in HIV prevention and detection behaviors (Ball et al., 2013). Indeed, Black people with HIV (PWH) evidence longer durations from HIV diagnosis to initiation of antiretroviral therapy (ART) and virologic suppression as compared to White PWH (Haines et al., 2016). Experiences of poverty, social stigmatization, and structural racism contribute to challenges in long-term HIV management in Black PWH (Freeman et al., 2020). In particular, discrimination and suboptimal management of mental health disorders (e.g., depression, substance use disorder) are associated with worse medical treatment adherence for Black PWH (Casagrande et al., 2007; Rawlings \& Masters, 2008). These multi-fold challenges for Black PWH have serious adverse consequences as evidenced by greater risk for medical co-morbidities (e.g., hepatitis $\mathrm{C}$ virus, renal and vascular disease; Rawlings \& Masters, 2008) and higher mortality rates than that of White and Hispanic PWH (Siddiqi et al., 2015). These health disparities highlight the need to better understand the many health challenges experienced by Black PWH.

Racial disparities are also evident in the neurocognitive health of Black Americans. Relative to White samples, Black Americans sometimes obtain lower scores on measures of verbal and visual learning and memory, executive functions, attention, and information processing speed, with effect sizes ranging from small to large (Heaton et al., 2004a; Manly et al., 2002; Norman et al., 2011; cf. Henderson et al., 1998; Strickland et al., 1997). Additionally, the relationship between sociodemographic factors and neurocognition may differ across race; for example, older age has been more strongly associated with lower neurocognitive performance among healthy Black versus White adults (Heaton et al., 2004a). 
Social and psychological phenomena including stereotype threat and perceived discrimination (e.g., Thames et al., 2013), socioeconomic disparities (e.g., Manly \& Echemendia, 2007), low education quality (e.g., Byrd et al., 2005), and acculturation (e.g., Manly et al., 2004) may partly explain the lower neuropsychological test scores sometimes observed among Black Americans. Most notably, lower literacy (i.e., proxy of education quality) is reliably associated with the score disparities in memory, language, and executive functions among Black Americans (Manly et al., 2005).

Taken together, the various sociodemographic, medical, and psychological factors that contribute to lower neurocognitive performance in Black Americans may interact with HIV disease to lead to worse brain health outcomes. For example, lower educational attainment and opportunities for Black Americans may decrease cognitive reserve (i.e., capacity to sustain cognitive disruption or compensate for losses using other cognitive regions; Stern, 2002), which can sometimes buffer against HIV-associated neurocognitive declines (Milanini et al., 2016; Vo et al., 2013). Oral word-reading is a commonly used proxy for cognitive reserve and can enhance diagnostic accuracy (i.e., in lieu of years of education for normative corrections) for detecting cognitive impairment in Black PWH (Rohit et al., 2007). Moreover, discrepancies between years of education and reading grade level are observed at higher rates in Black PLWH and are linked to lower neurocognitive scores (Ryan et al., 2005). Socially, experiences of adversity and lower acculturation for Black PWH may partially explain poorer outcomes in executive functions, processing speed, and episodic memory (Manly et al., 1998; Thames et al., 2018). Medical risk factors, including elevated rates of cardiovascular disease and viremia may also hasten the development of neurocognitive deficits among Black PWH (Storholm et al., 2019; Tedaldi et al., 2015). Furthermore, psychological disorders (e.g., depression) may exacerbate neurocognitive declines in Black Americans with medically symptomatic HIV (Richardson et al., 1999). Yet we still know little about the combined and unique effects of race and HIV on neuropsychological functioning.

Several published studies have examined the neuropsychological aspects of HIV disease in predominantly (e.g., Fogel et al., 2017) or exclusively (e.g., Durvasula et al., 2000) Black samples of PWH. However, such designs are limited in the extent to which they can dissect racial disparities for 
Black PWH. A smaller number of studies have examined the effects of race among PWH. Overall, those studies tend to show that Black PWH perform below White PWH (but comparable to Hispanic PWH) on measures of verbal fluency, information processing speed, executive functions, and working memory (Cruz et al., 2020; Marquine et al., 2016; Vo et al., 2013). For example, Winston and colleagues (2013) reported that Black PWH were over seven times more likely to meet Frascati-defined (Antinori et al., 2007) criteria for neurocognitive impairment than were White PWH. In a large exploratory study of 1,653 women with and without HIV, Manly and colleagues (2011) found that Black women scored lower than White women on measures of processing speed, executive functions, and word reading, irrespective of HIV serostatus. Thus, we can reliably assert that both race and HIV are associated with lower neurocognitive test scores, but it is unclear whether Black PWH experience an additive or synergistic burden of HIV disease on neurocognitive functioning.

Of course, a major motivation to understand racial disparities in neuropsychological functioning is its potential downstream implications for health behaviors and everyday functioning. Although functional declines have been examined in aging (e.g., Morgan et al., 2012) and neuropsychologically impaired (e.g., Heaton et al., 2004b) HIV populations, relatively little is known about ethnoracial differences among PWH. The literature suggests that Black PWH exhibit difficulties in medication management and adherence relative to their White counterparts (Patton et al., 2012; Thames et al., 2012). Waldrop-Valverde et al. (2010) reported that poorer medication management skills as measured in the laboratory were explained by lower numeracy in Black PWH. More broadly, greater declines in basic ADL domains (e.g., bathing, grooming) were observed in Black PWH who exhibited elevated biomarkers of allostatic load (i.e., lifetime and daily stressors; Fazeli et al. 2020). However, beyond these findings there is a dearth of research explicitly investigating the expression and neurocognitive correlates of everyday functional difficulties for Black PWH.

The current study investigated the individual and combined effects of race (i.e., Black versus White race) and HIV serostatus on neurocognitive outcomes across six domains. We hypothesized that Black PWH would show greater deficits in neurocognitive performance relative to White and 
seronegative individuals. In particular, we predicted that lower scores would be notable for Black PWH in declarative memory, executive functions, and processing speed based upon previous literature which suggests impairment in these domains for individuals with HIV and for those who identify as Black. A secondary aim was to examine differences in rates of neurocognitive disorders based on racial group and HIV serostatus. We predicted that higher rates of neurocognitive disorders would be observed in Black PWH. A third aim of this study was to investigate the ways in which neurocognition relates to clinical HIV biomarkers and everyday functioning within Black and White PWH. Based on prior work which established links between low health literacy and poorer HIV biomarkers within Black, but not White, PWH (Walker et al., 2018), we predicted that lower CD4 cell counts (nadir and current) and detectable viral loads would be more strongly associated with poorer neurocognitive functions for Black relative to White PWH. We also expected poorer neurocognitive functions to be associated with greater difficulties in everyday functioning for both HIV groups and a particularly strong association within Black PWH.

\section{Methods}

\section{Participants}

The sample included 40 Black PWH, 83 White PWH, 28 Black HIV- and 64 White HIV-adults aged 18 to 66 years. Participants were enrolled in a study of memory housed at the University of California San Diego (UCSD) HIV Neurobehavioral Research Program, which recruits from communitybased organizations, local clinics, and regional advertisements. Individuals were excluded from the study if they met any of the following criteria: history of psychotic disorders, intellectual disability, and major neuromedical conditions (e.g., active central nervous system opportunistic infections, head injury with loss of consciousness more than $30 \mathrm{~min}$, stroke with neurological sequelae, non-HIV-related dementias). In addition, participants were screened for substance use disorders (SUD) using the Diagnostic and Statistical Manual of Mental Disorders (4 ${ }^{\text {th }}$ ed.; American Psychiatric Association, 1994) and illicit drug use on the day of testing. Participants were excluded if they met criteria for current SUD or if they tested positive on a breathalyzer or urine toxicology screening on the day of testing (with marijuana as the exception). HIV serostatus was confirmed using Medmira or western blot test. All participants provided 
written, informed consent. Note that the current sample was derived from a larger parent study cohort described in Matchanova et al. (2020), Shepherd et al. (2020) and Tierney et al. (2017). Investigators for the current study were blinded to neurocognitive status of participants in the parent study cohort and extracted the four study groups (i.e., Black PWH, White PWH, Black HIV-, and White HIV-) on a casewise basis to be comparable in age, education, and Hollingshead scores. See Table 1 for relevant sociodemographic and health characteristics of the final study sample.

\section{Procedures}

Neurocognitive Functions

Participants were administered a comprehensive neuropsychological battery that assessed attention, executive functions, processing speed, episodic memory, semantic memory, and motor functions. Attention was measured using the Digit Span subtest of the Wechsler Adult Intelligence Scale, $3^{\text {rd }}$ edition (WAIS-III; Wechsler, 1997a), and Trial 1 of the California Verbal Learning Test-second edition (CVLT-II; Delis et al., 2000). Executive functions were assessed with the Total Moves score from the Tower of London Test (Drexel Version; Culbertson \& Zillmer, 1999), Action (verb) fluency test (Woods et al., 2005), and Trail Making Test, Part B (Army Individual Test Battery, 1944; Heaton et al., 2004a). Measures evaluating episodic memory included Logical Memory I and II from the Wechsler Memory Scale, 3rd edition (WMS-III; Wechsler, 1997b) and the Total Trials 1-5 and Long Delay Free Recall scores from the CVLT-II. Semantic memory was evaluated using the Boston Naming Test (BNT; Kaplan et al., 1983) and the Famous Faces subtest of the Kaufman Adolescent and Adult Intelligence Test (KAIT-FF; Kaufman \& Kaufman, 1993). Processing speed was assessed with Trail Making Test, Part A and execution time for the Tower of London Test. Lastly, motor functions were assessed via time to complete dominant and nondominant hands on the Grooved Pegboard Test (Heaton et al., 2004a; Kløve, 1963). For the primary analyses, the score for each subtest was converted to sample-based z-scores to allow comparison across study groups without the confounding influence of demographically-adjusted normative standards. Average sample-based z-scores were calculated for each neurocognitive domain based on the subtests detailed above. 


\section{Everyday Functioning}

Multiple domains of everyday functioning were also assessed in accordance with Frascati guidelines (Antinori et al., 2007). Self-reported daily functioning was examined with the Lawton \& Brody Activities of Daily Living Questionnaire - Heaton Revision (ADLQ; see Heaton et al., 2004a, b; Woods et al., 2004), which assessed functioning across 16 instrumental ADL domains at "best" and "now." The Karnofsky Performance Status Scale (KPSS; Karnofsky \& Burchenal, 1949) provided a clinical rating of participant functional status on a scale from 0 (dead) to 100 (normal activity). Self-reported employment status was gathered via semi-structured interview and coded into one of four levels: employed, retired, unemployed, or disabled. Everyday memory symptoms were evaluated using the 16-item Prospective and Retrospective Memory Questionnaire (PRMQ; Smith et al., 2000), which quantifies the frequency of memory concerns in daily life on a scale from 1 (never) to 5 (very often). Lastly, the 7-item Confusion and Bewilderment Scale from the Profile of Mood States (POMS; McNair et al., 1981) was used to measure perceived cognitive symptoms (e.g., unable to concentrate) over the past week on a scale from 0 (not at all) to 4 (extremely).

Each functional measure was used to determine functional status in line with Matchanova and colleagues' (2020) operationalization of the Frascati criteria (i.e., intact functioning, mild decline, or major decline). Additionally, each functional measure was coded such that "1" indicated functional decline (i.e., Frascati classification "mild" or "major" for that measure) and " 0 " indicated no functional decline. Then, a continuous global functional score was generated by summing these codes across the five functional measures for each participant whereby higher scores indicated a greater number of dependent functional domains (see Doyle et al., 2013).

\section{Classification of Neurocognitive Disorders}

The level of neurocognitive and functional impairment was assessed for each participant to classify HIV-associated neurocognitive disorders according to the Frascati criteria (Antinori et al., 2007; Woods et al., 2004). For clinical classification purposes, each raw neurocognitive subtest score was converted to a standardized T-score using normative data adjusting for age, education, sex, and race when 
available. Tests in the current battery with normative data that specifically corrected for race/ethnicity included BNT, Trails Making Test Parts A and B, and Grooved Pegboard. Cognitive impairment was determined using the Global Deficit Score (GDS) approach which assigns values from 0 (no impairment) to 5 (severe impairment) to the individual standardized T-scores, which are then averaged. Based upon these scores, cognitive functioning was dichotomized as either "impaired" or "within normal limits" using a cut-point of 0.5 (see Carey et al., 2004). Next, the GDS and Frascati functional diagnoses were both evaluated to classify neurocognitive disorders for each participant. For participants whose GDS was within normal limits, they were classified as neurocognitively "normal." Participants were classified with subsyndromic neurocognitive disorder when GDS was impaired and global Frascati functioning was intact. Syndromic neurocognitive disorders (e.g., mild neurocognitive disorder or HIV-associated dementia) were classified by impaired GDS and any level of global Frascati functional impairment (i.e., mild or major decline).

\section{Data Analysis}

The four study groups were comparable on age, education, SUD, and hepatitis C virus (HCV). A data-driven approach was used to select covariates (Field-Fote, 2019), whereby any sociodemographic variables in Table 1 that significantly related to both the independent and dependent variable in each model were included as covariates for that model. A multivariate analysis of variance (MANOVA) was utilized to examine main effects and interactions of HIV serostatus and race on sample-based z-scores across six cognitive domains. Planned post-hoc analyses were then conducted using a one-way analysis of variance (ANOVA) to compare neurocognitive domain scores across the four study groups. Any significant ANOVAs were followed up with all pairwise comparisons to examine specific group differences using the Tukey-Kramer HSD correction to reduce Type I error. A logistic regression was employed to determine whether HIV serostatus, race, or their interaction were associated with clinical classifications of neurocognitive disorders. Additionally, nonparametric Spearman's rho correlations were employed within the HIV groups to examine the relationship of global neurocognition with both HIV 
disease markers and everyday functioning separately within Black and White PWH. A critical alpha of .05 was used for all primary analyses.

\section{Results}

A MANOVA was conducted to examine the main effects and interactions of HIV serostatus and race across six neurocognitive domains, covarying for WTAR VIQ. The omnibus model was significant, $F(4,208)=24.86, p<.001, \eta^{2}=.48$, and revealed main between-subjects effects of HIV serostatus, $F(1,208)$ $=5.95, p=.016, \eta^{2}=.03$, and WTAR VIQ, $F(1,208)=66.48, p<.001, \eta^{2}=.32$. There was no main effect of race, $(p=.677)$ and the HIV by race interaction was not significant $(p=.729)$. A significant within-subjects effect of neurocognitive domain was also observed, $F(5,204)=5.28, p<.001, \eta^{2}=.13$. However, these main effects were tempered by a significant three-way interaction between HIV, race, and neurocognitive domain, $F(5,204)=3.23, p=.008, \eta^{2}=.08$.

Planned post-hoc analyses were then conducted using ANOVA and Tukey-Kramer HSD pairwise comparisons to compare the neurocognitive domain scores of the four study groups (see Figure 1). The domains of semantic memory $(F(3,214)=17.28, p<.001)$ and processing speed $(F(3,214)=3.19, p=$ .025) both showed significant omnibus effects that were driven by the Black PWH participants scoring lower than the White PWH and HIV-groups ( $p$ s $<.045$, mean Cohen's $d=.80)$. The domains of attention $(F(3,214)=8.02, p<.001)$ and episodic memory $(F(3,214)=7.80, p<.001)$ also showed significant omnibus effects, but these domain findings were characterized by higher scores in the White HIV-group as compared to both the White and Black PWH groups $(p \mathrm{~s}<.001$, mean $d=.74)$. There were no significant omnibus group differences observed in the domains of motor skills $(F(3,212)=1.03, p=.38)$ or executive functions $(F(3,214)=1.48, p=.22)$.

Next, we conducted a logistic regression to determine whether HIV serostatus, race, or their interaction were associated with clinical classifications of neurocognitive disorders. Lifetime affective disorder and WTAR VIQ were included as covariates in the model. The overall model was significant, $X^{2}(10, \mathrm{~N}=215)=48.59, p<.001$, and lifetime affective diagnosis $\left(X^{2}=10.06, p=.007\right)$ and WTAR VIQ 
$\left(X^{2}=18.10, p<.001\right)$ emerged as significant predictors. The race by HIV interaction term fell at the level of a trend $\left(X^{2}=5.64, p=.059\right)$. Post-hoc chi-square analyses were conducted in order to compare neurocognitive disorder classification rates between the study groups. Findings revealed greater rates of subsyndromic and syndromic neurocognitive disorder classifications for Black PWH participants compared to the other three groups ( $p s<.017$, mean Cramer's $V=.33$ ). Figure 2 displays the impairment classifications across the four study groups.

Finally, we examined the association between global neurocognition and both HIV disease markers and global everyday functioning in the PWH sample. Nonparametric correlations between HIV disease markers and global neurocognitive sample-based z-scores were all small (range $r_{s}=-.19$ to .22 ) and non-significant $(p s>.05)$ in both the Black and White PWH groups. The Spearman's rho correlation between global neurocognitive z-score and global functional impairment was significantly smaller $(z=$ $2.11, p=.017)$ in Black $\left(r_{s}=.01, p=.96\right)$ as compared to White $\left(r_{s}=-.39, p<.001\right)$ PWH (see Figure 3).

Discussion

Black Americans are at high risk for HIV disease and associated morbidity. However, we know little about the neuropsychological impact and correlates of HIV disease specifically among Black Americans. Using a full factorial design, the current study showed that HIV and race have synergistic adverse effects on some aspects of neuropsychological functioning. Specifically, we observed large effect size score disparities for Black PWH in the domains of semantic memory (i.e., naming objects and famous faces) and speeded information processing (i.e., visuomotor sequencing and visual planning speed). The score disparities for Black PWH in this study were not better explained by other sociodemographics (e.g., educational attainment, estimated VIQ), psychiatric factors, or medical comorbidities. Of particular importance in this regard was the inclusion of estimated VIQ (i.e., oral word reading) in these models, which prior studies show is an important modulator of the effects of race on neuropsychological test scores (e.g., Manly et al., 2004). Overall, these findings align with prior work showing lower scores in these neuropsychological domains for Black Americans with and without HIV disease (e.g., Vo et al., 2013), but extend that work by demonstrating the combined effects of these two 
risk factors. In other words, Black Americans may be at greater risk for experiencing the adverse effects of HIV disease on neuropsychological function and other aspects of brain health. The reasons for these brain health disparities in HIV remain to be determined by future research, which will require larger samples and the measurement of relevant constructs such as perceived racial stress, stereotype threat, education quality, and provider trust.

It is also important to highlight the several domains in which we did not observe the synergistic adverse effects of HIV and race in this study. First, the pattern of results for the domains of attention and episodic memory was unexpected. Specifically, both Black and White individuals with HIV performed worse than White seronegatives in these two domains, indicating there was no additive effect of HIV for Black participants. This was interesting considering that episodic memory is historically one of the most sensitive domains to HIV disease (e.g., Carey et al., 2004). Type II error is a possible explanation for this finding, as our sample of Black Americans was relatively small. Alternatively, prior findings link HIV and social adversity (e.g., low childhood SES, racial discrimination) to reduced volume in the amygdala and hippocampus, as well as poorer observed learning and memory performance (Thames et al., 2018). Perhaps in our sample both White and Black individuals experienced social adversity related to their HIV status, and thus revealed comparable performance in this domain. Null group-level differences were observed for motor skills and executive functions, such that Black and White individuals with and without HIV disease demonstrated comparable performance in these domains, after controlling for oral word reading. These findings were also surprising given that these domains are often impacted in HIV disease at small-to-medium effect sizes (Walker \& Brown, 2018; Woods et al., 2009). However, one study found the effect of HIV on executive functions was attenuated after accounting for race and educational factors (Manly et al., 2011). Taken together with our results, these findings indicate the importance of controlling for and investigating the roles of sociodemographic variables, and especially race, to elucidate the influences of HIV disease versus contextual factors on cognitive outcomes.

Next, we examined the clinical implications of these raw score findings by assessing whether race and HIV combined to impact rates of neurocognitive disorders in the sample. Even though several of the 
tests in this battery were normatively adjusted for race, which arguably increases our risk of Type II error, we nevertheless observed a modest, trend-level interaction between race and HIV serostatus on the frequency of neurocognitive disorders. Planned post-hoc analyses showed significantly higher rates of subsyndromic and syndromic neurocognitive disorders in Black individuals with HIV compared to the rest of the sample. Findings were associated with a medium effect size and were independent of critical covariates, including estimated VIQ. Nearly half of the Black PWH in our sample met criteria for a neurocognitive disorder, with half of those persons being classified as syndromic (i.e., exhibiting functional impairments). This observed frequency of HAND was aligned with neuroepidemiological estimates in the cART era (e.g., Heaton et al, 2010; Wang et al., 2020). Likewise, the rate of impairment in the seronegatives is reasonable for a comparison sample that includes persons with risk factors for neurocognitive impairment (e.g., depression, SUD, HCV). Oddly, the rate of HAND was lower than expected in the White $\mathrm{PWH}$, reasons for which are not entirely clear, but could be due to sampling bias in an urban Southern California community, or greater concurrence in quality and quantity of education for White PWH, which tends to influence better neurocognitive outcomes (e.g., Ryan et al., 2005). Additional explanations for the stark discrepancy in HAND rates for Black and White PWH might include healthcare discrimination that impacts HIV management (e.g., Casagrande et al., 2007), and potential influences of acculturation on neurocognitive assessments (e.g., BNT; Manly et al., 1998). At this juncture, however, these interpretations remain speculative. Future research is needed to explore the driving forces behind these discrepancies in rate of clinically significant impairment.

The diverging frequency of HAND was not driven by HIV disease severity for Black or White participants. Indeed, there was no relationship between HIV biomarkers with neurocognitive performance in either group and the accompanying effect sizes were small. Ultimately, the literature on HIV biomarkers and their association with neurocognitive outcomes across race/ethnicity is mixed and somewhat inconsistent. Although lower nadir CD4 counts are reliably linked to HIV-associated neurocognitive impairment (Ellis et al., 2011; Hassanzadeh-Behbahani et al., 2020; Wang et al., 2020) and brain volumes (Jernigan et al., 2011), the effect sizes tend to be small. In Black PWH, global 
neurocognition has been linked to current CD4 cell counts (Marquine et al. 2016). However, the same study found HIV RNA did not relate to neurocognition for either Black or White PWH. Domain specific findings reveal associations between viral load and poorer semantic memory (Fama et al., 2011) and motor functions (Lyons et al., 2011). Moreover, low current CD4 counts were linked to poorer language fluency and motor functions (Lyons et al., 2011), and reveal moderate associations with poorer health literacy for Black PWH specifically (Walker et al., 2018). In our current small HIV sample, we did not observe any meaningful relationships between HIV biomarkers and neurocognition for Black or White PWH. Future work might examine potential race differences in the association between cognition and other biomarkers relevant to HIV disease, including markers of inflammation, neural injury, astrocytosis, and vascular disease (Chan \& Brew, 2014).

A particularly intriguing finding from this study was that lower raw neuropsychological test scores were not associated with worse everyday functioning for Black PWH. The effect size was very small, null, and significantly lower than the medium association between cognition and everyday functioning that was observed in White PWH. The literature shows a robust, reliable relationship between poorer neuropsychological scores and many aspects of everyday functioning in HIV disease (Heaton et al., 2004b), which raises the question as to why this same relationship was not present in this sample of Black PWH. Again, issues of Type II error and sampling are possible, but unsatisfactory explanations. Prior studies suggest that cognitive activity (e.g., read newspaper, attend concert, visit museum) may play a uniquely strong role in the brain health of Black PWH (Krueger et al., 2017), which can affect everyday functions (e.g., Morgan et al., 2012). It is also possible that other barriers that are unique to (or amplified in) Black PWH could interfere with health behaviors and everyday functioning, including structural barriers (e.g., financial, legal), food insecurity, social adversity (e.g., institutional racism, social isolation), and HIV stigma (Freeman et al., 2020; Watson et al., 2019). From a clinical standpoint, it is important to note that functional impairment may present itself prior to and independent of neurocognitive deficits, and perhaps in the current study we observed this uniquely within Black PWH (Heaton et al., 2004b; Kraal et al., in press). Finally, we might consider how positive psychological factors, like resilience and wisdom, 
could provide some everyday functioning protection from neurocognitive impairment in Black PWH (Moore et al., 2018). These hypothesized explanations inspire future investigations which are required to understand the clinical presentation of Black PWH in order to target their specific cognitive and functional needs.

This study has several limitations that should be considered in interpreting and applying its findings. First, the sample is largely middle-aged men from urban areas in Southern California and may not be representative of Black Women, older adults, and/or people from highly affected areas (e.g., the Southeast U.S. or Africa). Second, this study did not include any neuroimaging, neuropathology, or brain biomarkers (e.g., tau), which will be important for future studies of brain health disparities in Black PWH. Also, we did not assess some important socioeconomic and cultural variables, nor did we include measures of perceived or experienced racial discrimination which may illuminate possible explanations for the negative cognitive outcomes we observed in Black PWH. Moreover, health literacy may be another important contributing variable that could have reciprocal effects on neurocognitive and health outcomes in HIV (Walker et al., 2018). To assess everyday functioning, we included clinician-rated and self-report measures, all of which are subject to reporter biases and may not accurately capture functional capacity for individuals whose insight into actual functioning is limited. Studies using performance-based measures of everyday functioning (e.g., financial skills) and health behaviors (e.g., medication management) will be important in diverse populations with HIV. Future work could also examine Internet-based disparities, since Black PWH may have difficulty with access (e.g., Mayben \& Giordano, 2007) and performance (Matchanova et al., in press) on these increasingly common tools for daily living. Despite those limitations, findings from this study point to fundamental brain health disparities for Black PWH that warrant the attention of clinicians and scientists. At the level of inclusion, we need to be conscious of using modern community engagement efforts to recruit, enroll, and retain Black PWH and at-risk seronegatives in our research to ensure representativeness (Dancy et al., 2004; Gwadz et al., 2014). We also need to actively examine possible main effects and interactions with race at all levels of analysis in neuroAIDS studies. Too often race is treated as a "nuisance" variable to be dismissed as a 
confound, rather than being considered as a primary variable of scientific interest. Our findings reveal the existence of racial differences for cognitive health outcomes in Black PWH that require our attention to fully understand the scope of these effects. Taken a step further, we also need to develop cognitive rehabilitation strategies that may enhance neurocognitive performance in Black PWH and reduce disparities in brain health for this population. Intervention studies should consider the role of race and the possibility that existing and/or novel strategies may show different efficacy and effectiveness for Black PWH. Based upon the domains in which we observed synergistic effects of HIV and race, we can recommend increasing cognitive reserve (e.g., by using spaced-retrieval practice; Avci et al., 2017) and training speed of processing (e.g., Vance et al. 2012) as starting points for cognitive interventions for Black PWH.

\section{Acknowledgements and Disclosure Statement}

This work was supported by the National Institutes of Health under grants R01-MH73419 and P30-MH62512. The authors thank the University of California, San Diego HIV Neurobehavioral Research Program (HNRP) Group (I. Grant, PI) for their infrastructure support of the parent R01. In particular, we are grateful to Donald Franklin, Dr. Erin Morgan, Clint Cushman, and Stephanie Corkran for their assistance with data processing, Marizela Verduzco for her assistance with study management, Dr. Scott Letendre and Dr. Ronald J. Ellis for their assistance with the neuromedical aspects of the parent project, and Dr. J. Hampton Atkinson and Jennifer Marquie Beck for their assistance with participant recruitment and retention. The views written in this article are those of the authors and do not reflect the official policy or position of the Department of the Navy, Department of Defense, nor the United States Government. The authors would also like to thank the study volunteers for their participation. 


\section{References}

American Psychiatric Association (APA). (1994). Diagnostic and statistical manual of mental disorders ( $4^{\text {th }}$ ed.). Arlington, VA: American Psychiatric Publishing, Inc.

Antinori, A., Arendt, G., Becker, J. T., Brew, B. J., Byrd, D. A., Cherner, M., Clifford, D. B., Cinque, P., Epstein, L. G., Goodkin, K., Gisslen, M., Grant, I., Heaton, R. K., Joseph, J., Marder, K., Marra, C. M., McArthur, J. C., Nunn, M., Price, R.W. ... Wojna, V. E. (2007). Updated research nosology for HIV-associated neurocognitive disorders. Neurology, 69(18), 1789-1799.

Army, U. S. (1944). Army individual test battery. Manual of directions and scoring.

Avci, G., Woods, S. P., Verduzco, M., Sheppard, D. P., Sumowski, J. F., Chiaravalloti, N., \& DeLuca, J. (2017). The Effect of Retrieval Practice on Short-Term and Long-Term Retention in HIV+ Individuals. Journal of the International Neuropsychological Society : JINS, 23(3), 214222. https://doi.org/10.1017/S1355617716001089

Ball, K., Lawson, W. L., \& Alim, T. (2013) Medical mistrust, conspiracy beliefs and HIV-related behavior among African Americans. Journal of Psychology and Behavioral Science, 1(1), 1-7.

Blankenship, K. M., Smoyer, A. B., Bray, S. J., \& Mattocks, K. (2005). Black-white disparities in HIV/AIDS: the role of drug policy and the corrections system. Journal of health care for the poor and underserved, 16(4 Suppl B), 140-156. https://doi.org/10.1353/hpu.2005.0110

Brooks, J. R., Hong, J. H., Cheref, S., \& Walker, R. L. (2020). Capability for suicide:

Discrimination as a painful and provocative event. Suicide and Life-threatening Behavior, 00, 18. http://doi.org/10.1111/sltb.12671

Byrd, D. A., Sanchez, D., \& Manly, J. J. (2005). Neuropsychological test performance among Caribbean-born and U.S.-born African American elderly: The role of age, education and reading level. Journal of Clinical and Experimental Neuropsychology, 27(8), 1056-1069. http://doi.org/10.1080/13803390490919353

Carey, C. L., Woods, S. P., Gonzalez, R., Conover, E., Marcotte, T. D., Grant, I., \& Heaton, R. K. (2004). Predictive validity of Global Deficit Scores in detecting neuropsychological impairment in HIV 
infection. Journal of Clinical and Experimental Neuropsychology, 26(3), 307-319. doi:10.1080/13803390490510031

Casagrande, S. S., Gary, T. L., LaVeist, T. A., Gaskin, D. J., \& Cooper, L. A. (2007). Perceived discrimination and adherence to medical care in a racially integrated community. Journal of general internal medicine, 22(3), 389-395. https://doi.org/10.1007/s11606-006-0057-4

Centers for Disease Control (CDC) and Prevention. (2020). Diagnoses of HIV Infection in the United States and Dependent Areas HIV Surveillance Report 2018 (updated),

31. http://www.cdc.gov/hiv/library/reports/hiv-surveillance.html.

Chan, P., \& Brew, B. J. (2014). HIV associated neurocognitive disorders in the modern antiviral treatment era: prevalence, characteristics, biomarkers, and effects of treatment. Current HIV/AIDS Reports, 11(3), 317-324.

Cruz, L. N., Weinberger, A. H., Shuter, J., \& Lee, C. J. (2020). Wisconsin Card Sorting Task-64 performance among HIV+ Black/African American and Latinx adults compared to normative samples and by sociocultural and health variables. Applied Neuropsychology: Adult, 1-13. https://doi.org/10.1080/23279095.2020.1813142

Culbertson, W. C., \& Zillmer, E. A. (1999). The Tower of London, Drexel University, research version: examiner's manual. North Tonawanda: Multi-Health Systems.

Dancy, B. L., Wilbur, J., Talashek, M., Bonner, G., \& Barnes-Boyd, C. (2004). Communitybased research: Barriers to recruitment of African Americans. Nursing Outlook, 52(5), 234-240. https://doi.org/10.1016/j.outlook.2004.04.012

Delis, D. C., Kramer, J. H., Kaplan, E., \& Ober, B. A. (2000). California Verbal Learning Test-Second Edition (CVLT-II). San Antonio, TX: The Psychological Corporation.

Doyle, K. L., Morgan, E. E., Morris, S., Smith, D. M., Little, S., Iudicello, J. E., Blackstone, K., Moore, D. J., Grant, I., Letendre, S. L., Woods, S. P., \& Translational Methamphetamine AIDS Research Center (TMARC) Group (2013). Real-world impact of neurocognitive deficits in acute 
and early HIV infection. Journal of neurovirology, 19(6), 565-573.

https://doi.org/10.1007/s13365-013-0218-2

Durvasula, R. S., Myers, H. F., Satz, P., Miller, E. N., Morgenstern, H., Richardson, M. A., Evans, G., \& Forney, D. (2000). HIV-1, cocaine, and neuropsychological performance in African American men. Journal of the International Neuropsychological Society, 6(3), 322-335. https://doi.org/10.1017/S1355617700633076

Ellis RJ, Badiee J, Vaida F, et al. CD4 nadir is a predictor of HIV neurocognitive impairment in the era of combination antiretroviral therapy. AIDS. 2011;25(14):1747-1751. https://doi.org/10.1097/QAD.0b013e32834a40cd

Fama, R., Rosenbloom, M. J., Sassoon, S. A., Thompson, M. A., Pfefferbaum, A., \& Sullivan, E. V. (2011). Remote semantic memory for public figures in HIV infection, alcoholism, and their comorbidity. Alcoholism: Clinical and Experimental Research, 35(2), 265-276.

Fazeli, P. L., Waldrop-Valverde, D., Yigit, I., Turan, B., Edberg, J., Kempf, M., \& Vance, D. (2020). An Exploratory Study of Correlates of Allostatic Load in Older People Living With HIV: JAIDS Journal of Acquired Immune Deficiency Syndromes, 83(5), 441-449. https://doi.org/10.1097/QAI.0000000000002293

Field-Fote, E. (2019). Mediators and moderators, confounders and covariates: exploring the variables that illuminate or obscure the "active ingredients" in neurorehabilitation. Journal of Neurologic Physical Therapy, 43(2), 83-84. https://doi.org/10.1097/NPT.0000000000000275.

Fogel, J., Rubin, L. H., Maki, P., Keutmann, M. K., Gonzalez, R., Vassileva, J., \& Martin, E. M. (2017). Effects of sex and HIV serostatus on spatial navigational learning and memory among cocaine users. Journal of NeuroVirology, 23(6), 855-863. https://doi.org/10.1007/s13365-0170563-7

Freeman, R., Gwadz, M., Wilton, L., Collins, L. M., Dorsen, C., Hawkins, R. L., Silverman, E., Martinez, B. Y., Leonard, N. R., Applegate, A., \& Cluesman, S. (2020). Understanding long-term HIV survivorship among African American/Black and Latinx persons living with HIV in the 
United States: A qualitative exploration through the lens of symbolic violence. International Journal for Equity in Health, 19(1), 146. https://doi.org/10.1186/s12939-020-01253-w

Gwadz, M., Cleland, C. M., Belkin, M., Ritchie, A., Leonard, N., Riedel, M., Banfield, A., Colon, P., Elharrar, V., Kagan, J., \& Mildvan, D. (2014). ACT2 peer-driven intervention increases enrollment into HIV/AIDS medical studies among African-Americans/Blacks and Hispanics: A cluster randomized controlled trial. AIDS and Behavior, 18(12), 2409-2422. https://doi.org/10.1007/s10461-014-0829-5

Haines, C. F., Fleishman, J. A., Yehia, B. R., Lau, B., Berry, S. A., Agwu, A. L., Moore, R. D., \& Gebo, K. A. (2016). Closing the gap in antiretroviral initiation and viral suppression: Time trends and racial disparities. Journal of Acquired Immune Deficiency Syndromes (1999), 73(3), 340-347. https://doi.org/10.1097/QAI.0000000000001114

Hassanzadeh-Behbahani, S., Shattuck, K. F., Bronshteyn, M., Dawson, M., Diaz, M., Kumar, P., Moore, D. J., Ellis, R. J., \& Jiang, X. (2020). Low CD4 nadir linked to widespread cortical thinning in adults living with HIV. NeuroImage: Clinical, 25, 102155. https://doi.org/10.1016/j.nicl.2019.102155

Heaton, R. K., Marcotte, T. D., Mindt, M. R., Sadek, J., Moore, D. J., Bentley, H., Mccutchan, J. A., Reicks, C., Grant, I., \& THE HNRC GROUP. (2004b). The impact of HIV-associated neuropsychological impairment on everyday functioning. Journal of the International Neuropsychological Society, 10(03). https://doi.org/10.1017/S1355617704102130

Heaton, R. K., Miller, S. W., Taylor, M. J., \& Grant, I. (2004a). Revised comprehensive norms for an expanded Halstead-Reitan Battery: Demographically adjusted neuropsychological norms for African American and Caucasian adults. Lutz, FL: Psychological Assessment Resources.

Henderson, L. W., Frank, E. M., Pigatt, T., Abramson, R. K., \& Houston, M. (1998). Race, gender, and educational level effects on Boston Naming Test scores. Aphasiology, 12(10), 901911. http://doi.org/10.1080/02687039808249458

Jernigan, T. L., Archibald, S. L., Fennema-Notestine, C., Taylor, M. J., Theilmann, R. J., Julaton, M. 
D., Notestine R. J., Wolfson T., Letendre S. L., Ellis R. J., Heaton R. K., Gamst A. C., Franklin, D. R. Jr., Clifford, D. B., Collier, A. C., Gelman, B. B., Marra, C., McArthur, J. C., McCutchan, J. A., Morgello, S., Simpson, D. M., Grant, I., \& The CHARTER Group. (2011). Clinical factors related to brain structure in HIV: the CHARTER study. Journal of neurovirology, 17(3), 248. https://doi.org/10.1007/s13365-011-0032-7.

Kaplan, E., Goodglass, H., \& Weintraub, S. (1983). Boston Naming Test. Philadelphia: Lea \& Febiger.

Karnofsky, D. A. \& Burchenal, J. H. (1949). The clinical evaluation of chemotherapeutic agents in cancer. In: MacLeod, C.M., Ed., Evaluation of Chemotherapeutic Agents. Columbia University Press, New York, 191-205.

Kløve, H. (1963). Grooved pegboard. Lafayette, IN: Lafayette Instruments.

Kraal, A. Z., Massimo, L., Fletcher, E., Carrion, C. I., Medina, L. D., Mungas, D., Gavett, B. E., \& Farias, S. T. (in press). Functional reserve: The residual variance in instrumental activities of daily living not explained by brain structure, cognition, and demographics. Neuropsychology.

Krueger, K. R., Adeyemi, O., Leurgans, S., Shah, R. C., Jimenez, A. D., Ouellet, L., Landay, A. L., Bennett, D. A., \& Barnes, L. L. (2017). Association of cognitive activity and neurocognitive function in blacks and whites with HIV. AIDS (London, England), 31(3), 437-441. https://doi.org/10.1097/QAD.0000000000001316

Kaufman, A. S., \& Kaufman, N. L., (1993). Manual: Kaufman Adolescent and Adult Intelligence Test. Circle Pines, MN: American Guidance Service.

Lyons, J. L., Uno, H., Ancuta, P., Kamat, A., Moore, D. J., Singer, E. J., Morgello, S., \& Gabuzda, D. (2011). Plasma sCD14 is a biomarker associated with impaired neurocognitive test performance in attention and learning domains in HIV infection. JAIDS Journal of Acquired Immune Deficiency Syndromes, 57(5), 371-379. https://doi.org/10.1097/QAI.0b013e3182237e54

Manly, J. J., \& Echemendia, R. J. (2007). Race-specific norms: Using the model of hypertension to understand issues of race, culture, and education in neuropsychology. Archives of Clinical Neuropsychology, 22(3), 319-325. 
Manly, J. J., Byrd, D. A., Touradji, P., \& Stern, Y. (2004). Acculturation, reading level, and neuropsychological test performance among African American elders. Applied Neuropsychology, 11(1), 37-46.

Manly, J. J., Jacobs, D. M., Touradji, P., Small, S. A., \& Stern, Y. (2002). Reading level attenuates differences in neuropsychological test performance between African American and White elders. Journal of the International Neuropsychological Society: JINS, 8(3), 341-348. https://doi.org/10.1017/s1355617702813157

Manly, J. J., Miller, S. W., Heaton, R. K., Byrd, D., Reilly, J., Velasquez, R. J., Saccuzzo, D. P., Grant, I., \& The HIV Neurobehavioral Research Center (HNRC) Group. (1998). The effect of African-American acculturation on neuropsychological test performance in normal and HIVpositive individuals. Journal of the International Neuropsychological Society, 4(3), 291-302. doi:10.1017/S1355617798002914

Manly, J. J., Schupf, N., Tang, M. X., \& Stern, Y. (2005). Cognitive decline and literacy among ethnically diverse elders. Journal of geriatric psychiatry and neurology, 18(4), 213-217.

Manly, J. J., Smith, C., Crystal, H. A., Richardson, J., Golub, E. T., Greenblatt, R., Robison, E., Martin, E. M., \& Young, M. (2011). Relationship of ethnicity, age, education, and reading level to speed and executive function among HIV+ and HIV- women: the Women's Interagency HIV Study (WIHS) Neurocognitive Substudy. Journal of clinical and experimental neuropsychology, 33(8), 853-863. https://doi.org/10.1080/13803395.2010.547662

Marquine, M. J., Sakamoto, M., Dufour, C., Rooney, A., Fazeli, P., Umlauf, A., Gouaux, B., Franklin, D., Ellis, R., Letendre, S., Cherner, M., Heaton, R. K., Grant, I., \& Moore, D. J. (2016). The impact of ethnicity/race on the association between the Veterans Aging Cohort Study (VACS) Index and neurocognitive function among HIV-infected persons. Journal of Neurovirology, 22(4), 442-454. https://doi.org/10.1007/s13365-015-0411-6

Matchanova, A., Woods, S. P., Cushman, C., Morgan, E. E., Medina, L. D., Babicz, M. A., 
Verduzco, M. \& Loft, S. (in press). Online pharmacy navigation skills are associated with prospective memory in HIV disease. The Clinical Neuropsychologist.

Matchanova, A., Woods, S. P., \& Kordovski, V. M. (2020). Operationalizing and evaluating the Frascati criteria for functional decline in diagnosing HIV-associated neurocognitive disorders in adults. Journal of neurovirology, 26(2), 155-167. https://doi.org/10.1007/s13365-019-00809-z

Mayben, J. K., \& Giordano, T. P. (2007). Internet use among low-income persons recently diagnosed with HIV infection. AIDS care, 19(9), 1182-1187.

Mays, V. M., Cochran, S. D., \& Barnes, N. W. (2007). Race, race-based discrimination, and health outcomes among African Americans. Annual Review of Psychology, 58, 201-225. http://doi.org/10.1146/annurev.psych.57.102904.190212

McNair, D. M., Lorr, M., \& Droppleman, L. F. (1981). Profile of mood states manual. San Diego, CA: EDITS manual.

Milanini, B., Ciccarelli, N., Fabbiani, M., Limiti, S., Grima, P., Rossetti, B., Visconti, E., Tamburrini, E., Cauda, R., \& Di Giambenedetto, S. (2016). Cognitive reserve and neuropsychological functioning in older HIV-infected people. Journal of neurovirology, 22(5), 575-583. https://doi.org/10.1007/s13365-016-0426-7

Moore, D. J., Fazeli, P. L., Moore, R. C., Woods, S. P., Letendre, S. L., Jeste, D. V., \& Grant, I. (2018). Positive Psychological Factors Are Linked to Successful Cognitive Aging Among Older Persons Living with HIV/AIDS. AIDS and Behavior, 22(5), 1551-1561. https://doi.org/10.1007/s10461-017-2001-5

Morgan, E. E., Iudicello, J. E., Weber, E., Duarte, N. A., Riggs, P. K., Delano-Wood, L., Ellis, R., Grant, I., \& Woods, S. P. (2012). Synergistic Effects of HIV Infection and Older Age on Daily Functioning. Journal of Acquired Immune Deficiency Syndromes (1999), 61(3), 341-348. https://doi.org/10.1097/QAI.0b013e31826bfc53

Norman, M. A., Moore, D. J., Taylor, M., Franklin, D., Jr, Cysique, L., Ake, C., Lazarretto, D., 
Vaida, F., Heaton, R. K., \& HNRC Group (2011). Demographically corrected norms for African Americans and Caucasians on the Hopkins Verbal Learning Test-Revised, Brief Visuospatial Memory Test-Revised, Stroop Color and Word Test, and Wisconsin Card Sorting Test 64-Card Version. Journal of clinical and experimental neuropsychology, 33(7), 793-804. https://doi.org/10.1080/13803395.2011.559157

Patton, D. E., Woods, S. P., Franklin, D., Jr, Cattie, J. E., Heaton, R. K., Collier, A. C., Marra, C., Clifford, D., Gelman, B., McArthur, J., Morgello, S., Simpson, D., McCutchan, J. A., \& Grant, I. (2012). Relationship of Medication Management Test-Revised (MMT-R) performance to neuropsychological functioning and antiretroviral adherence in adults with HIV. AIDS and Behavior, 16(8), 2286-2296. https://doi.org/10.1007/s10461-012-0237-7

Rawlings, M. K., \& Masters, H. L. III. (2008). Comorbidities and challenges affecting African Americans with HIV infection. Journal of the National Medical Association, 100(12), 1477-1481. https://doi.org/10.1016/S0027-9684(15)31550-9

Richardson, M. A., Satz, P., Myers, H. F., Miller, E. N., Bing, E. G., Fawzy, F. I., \& Maj, M. (1999). Effects of Depressed Mood versus Clinical Depression on Neuropsychological Test Performance among African American Men Impacted by HIV/AIDS. Journal of Clinical and Experimental Neuropsychology, 21(6), 769-783. https://doi.org/10.1076/jcen.21.6.769.860

Rilling, L. M., Lucas, J. A., Ivnik, R. J., Smith, G. E., Willis, F. B., Ferman, T. J., et al. (2005). Mayo's Older African American Normative Studies: Norms for the Mattis Dementia Rating Scale. The Clinical Neuropsychologist, 19(2), 229-242.

Rohit, M., Levine, A., Hinkin, C., Abramyan, S., Saxton, E., Valdes-Sueiras, M., \& Singer, E. (2007). Education correction using years in school or reading grade-level equivalent? Comparing the accuracy of two methods in diagnosing HIV-associated neurocognitive impairment. Journal of the International Neuropsychological Society, 13(3), 462-470. https://doi.org/10.1017/S1355617707070506

Ryan, E. L., Baird, R., Mindt, M. R., Byrd, D., Monzones, J., Morgello, S., \& Manhattan HIV 
Brain Bank. (2005). Neuropsychological impairment in racial/ethnic minorities with HIV infection and low literacy levels: Effects of education and reading level in participant characterization. Journal of the International Neuropsychological Society, 11(7), 889.

Sheppard, D.P., Matchanova, A., Sullivan, K.L., Ishtiaq Kazimi, S., \& Woods, S.P. (2020).

Prospective memory partially mediates the association between aging and everyday functioning. The Clinical Neuropsychologist, 34, 755-774.

Siddiqi, A. E., Hu, X., Hall, H. I., \& Centers for Disease Control and Prevention (CDC) (2015). Mortality among blacks or African Americans with HIV infection--United States, 20082012. MMWR. Morbidity and mortality weekly report, 64(4), 81-86.

Smith, G., Della Sala, S., Logie, R.H., \& Maylor, E. A. (2000). Prospective and retrospective memory in normal ageing and dementia: A questionnaire study. Memory, 8, 311-321.

Steele, C. M., \& Aronson, J. (1995). Stereotype threat and the intellectual test performance of African Americans. Journal of Personality and Social Psychology, 69(5), 797-811. https://doi.org/10.1037/0022-3514.69.5.797

Stern, Y. (2002). What is cognitive reserve? Theory and research application of the reserve concept. Journal of the International Neuropsychological Society, 8(3), 448-460.

Storholm, E. D., Bogart, L. M., Mutchler, M. G., Klein, D. J., Ghosh-Dastidar, B., McDavitt, B., \& Wagner, G. J. (2019). Antiretroviral Adherence Trajectories Among Black Americans Living with HIV. AIDS and behavior, 23(8), 1985-1997. https://doi.org/10.1007/s10461-018-2303-2

Strickland, T. L., D'Elia, L. F., James, R., \& Stein, R. (1997). Stroop color-word performance of African Americans. The Clinical Neuropsychologist, 11(1), 87-90. http://doi.org/10.1080/13854049708407034

Tedaldi, E. M., Minniti, N. L., \& Fischer, T. (2015). HIV-associated neurocognitive disorders: The relationship of HIV infection with physical and social comorbidities. BioMed Research International, 641913. https://doi.org/10.1155/2015/641913

Thames, A. D., Hinkin, C. H., Byrd, D. A., Bilder, R. M., Duff, K. J., Mindt, M. R., Arentoft, A., 
\& Streiff, V. (2013). Effects of stereotype threat, perceived discrimination, and examiner race on neuropsychological performance: simple as black and white? Journal of the International Neuropsychological Society, 19(5), 583-593. https://doi.org/10.1017/S1355617713000076

Thames, A. D., Kuhn, T. P., Mahmood, Z., Bilder, R. M., Williamson, T. J., Singer, E. J., \& Arentoft, A. (2018). Effects of social adversity and HIV on subcortical shape and neurocognitive function. Brain Imaging and Behavior, 12(1), 96-108. https://doi.org/10.1007/s11682-017-96760

Thames, A. D., Moizel, J., Panos, S. E., Patel, S. M., Byrd, D. A., Myers, H. F., Wyatt, G. E., \& Hinkin, C. H. (2012). Differential predictors of medication adherence in HIV: Findings from a sample of African American and Caucasian HIV-positive drug-using adults. AIDS Patient Care and STDs, 26(10), 621-630. https://doi.org/10.1089/apc.2012.0157

Tierney, S. M., Sheppard, D. P., Kordovski, V. M., Faytell, M. P., Avci, G., \& Woods, S. P. (2017). A comparison of the sensitivity, stability, and reliability of three diagnostic schemes for HIV-associated neurocognitive disorders. Journal of neurovirology, 23(3), 404-421. https://doi.org/10.1007/s13365-016-0510-z

Vance, D. E., Fazeli, P. L., Ross, L. A., Wadley, V. G., \& Ball, K. K. (2012). Speed of processing training with middle-age and older adults with HIV: a pilot study. The Journal of the Association of Nurses in AIDS Care: JANAC, 23(6), 500-510. https://doi.org/10.1016/j.jana.2012.01.005

Vo, Q. T., Cox, C., Li, X., Jacobson, L. P., McKaig, R., Sacktor, N., Selnes, O. A., Martin, E., Becker, J. T., \& Miller, E. N. (2013). Neuropsychological test performance before and after HIV1 seroconversion: The Multicenter AIDS Cohort Study. Journal of NeuroVirology, 19(1), 24-31. https://doi.org/10.1007/s13365-012-0136-8

Waldrop-Valverde, D., Osborn, C. Y., Rodriguez, A., Rothman, R. L., Kumar, M., \& Jones, D. L. (2010). Numeracy Skills Explain Racial Differences in HIV Medication Management. AIDS and Behavior, 14(4), 799-806. https://doi.org/10.1007/s10461-009-9604-4 
Walker, K. A., \& Brown, G. G. (2018). HIV-associated executive dysfunction in the era of modern antiretroviral therapy: A systematic review and meta-analysis. Journal of Clinical and Experimental Neuropsychology, 40(4), 357-376. https://doi.org/10.1080/13803395.2017.1349879

Walker, R. L., Hong, J. H., Talavera, D. C., Verduzco, M., \& Woods, S. P. (2018). Health literacy and current CD4 cell count in a multiethnic U.S. sample of adults living with HIV infection. International Journal of STD \& AIDS, 29(5), 498-504. https://doi.org/10.1177/0956462417738679

Wang, Y., Liu, M., Lu, Q., Farrell, M., Lappin, J. M., Shi, J., Lu, L. \& Bao, Y. (2020). Global prevalence and burden of HIV-associated neurocognitive disorder: a metaanalysis. Neurology, 95(19), e2610-e2621.

Watson, C. W.-M., Sundermann, E. E., Hussain, M. A., Umlauf, A., Thames, A. D., Moore, R. C., Letendre, S. L., Jeste, D. V., Morgan, E. E., \& Moore, D. J. (2019). Effects of trauma, economic hardship, and stress on neurocognition and everyday function in HIV. Health Psychology: Official Journal of the Division of Health Psychology, American Psychological Association, 38(1), 33-42. https://doi.org/10.1037/hea0000688

Wechsler, D. (1997a). Wechsler Adult Intelligence Scale-Third edition. San Antonio, TX: Psychological Corporation.

Wechsler, D. (1997b). Wechsler Memory Scale-Third edition. San Antonio, TX: Psychological Corporation.

Williams, J. K., Ramamurthi, H. C., Manago, C., \& Harawa, N. T. (2009). Learning from successful interventions: A culturally congruent HIV risk-reduction intervention for African American men who have sex with men and women. American Journal of Public Health, 99(6), 1008-1012.

Winston, A., Arenas-Pinto, A., Stöhr, W., Fisher, M., Orkin, C. M., Aderogba, K., Burgh- 
Thomas, A. D., O’Farrell, N., Lacey, C. J. N., Leen, C., Dunn, D., \& Paton, N. I. (2013).

Neurocognitive Function in HIV Infected Patients on Antiretroviral Therapy. PLOS ONE. PLOS

ONE. http://doi.org/10.1371/journal.pone.0061949

Woods, S. P., Moore, D. J., Weber, E., \& Grant, I. (2009). Cognitive neuropsychology of HIVassociated neurocognitive disorders. Neuropsychology Review, 19(2), 152-168. https://doi.org/10.1007/s11065-009-9102-5

Woods, S. P., Rippeth, J. D., Frol, A. B., Levy, J. K., Ryan, E., Soukup, V. M., Hinkin, C. H., Lazzaretto, D., Cherner, M., Marcotte, T. D., Gelman, B. B., Morgello, S., Singer, E. J., Grant, I., \& Heaton, R. K. (2004). Interrater reliability of clinical ratings and neurocognitive diagnoses in HIV. Journal of Clinical and Experimental Neuropsychology, 26(6), 759-778. https://doi.org/10.1080/13803390490509565

Woods, S. P., Scott, J. C., Sires, D. A., Grant, I., Heaton, R. K., Tröster, A. I., \& HIV Neurobehavioral Research Center Group (2005). Action (verb) fluency: Test-retest reliability, normative standards, and construct validity. Journal of the International Neuropsychological Society, 11(4), 408-415. 
Table 1. Demographic and clinical characteristics of the four study groups.

\begin{tabular}{|c|c|c|c|c|c|}
\hline Variable & $\begin{array}{l}\text { White HIV- } \\
\quad(\mathrm{n}=64)\end{array}$ & $\begin{array}{l}\text { White HIV+ } \\
\qquad(\mathrm{n}=83)\end{array}$ & $\begin{array}{l}\text { Black HIV- } \\
(\mathrm{n}=28)\end{array}$ & $\begin{array}{l}\text { Black HIV+ } \\
\quad(\mathrm{n}=40)\end{array}$ & $p$ \\
\hline \multicolumn{6}{|l|}{ Sociodemographics } \\
\hline Age (years) & $44.5(14.4)$ & $44.9(10.4)$ & $43.4(13.9)$ & $39.7(12.0)$ & .157 \\
\hline Gender ( $\%$ men $)$ & 76.6 & 92.8 & 60.7 & 77.5 & $<.001$ \\
\hline Handedness (\% right) & 93.8 & 91.6 & 89.3 & 90.0 & .867 \\
\hline Education (years) & $13.7(2.2)$ & $13.2(2.2)$ & $13.6(1.9)$ & $12.8(1.9)$ & .117 \\
\hline GDS (\% impaired) & 18.8 & 16.9 & 14.3 & 45.0 & .004 \\
\hline Hollingshead highest occupation & $4.9(1.9)^{1}$ & $4.7(1.8)^{2}$ & $4.6(1.9)^{3}$ & $4.3(1.7)^{4}$ & .447 \\
\hline \multicolumn{6}{|l|}{ Psychiatric } \\
\hline POMS total (of 200) & $19.4(26.5)^{1}$ & $38.4(40.8)^{2}$ & $18.4(23.9)^{3}$ & $47.9(49.4)^{4}$ & $<.001$ \\
\hline \multicolumn{6}{|l|}{ Major depressive disorder $(\%)$} \\
\hline Current & 3.1 & $21.0^{2}$ & 7.1 & 10.0 & .006 \\
\hline $\begin{array}{l}\text { Lifetime } \\
\text { Generalized anxiety disorder }(\%)\end{array}$ & 39.1 & $72.8^{2}$ & 35.7 & 55.0 & $<.001$ \\
\hline Current & 0.0 & $8.6^{2}$ & 0.0 & 2.5 & .011 \\
\hline Lifetime & 1.6 & $21.0^{2}$ & 0.0 & 10.0 & $<.001$ \\
\hline Substance use disorder $(\%)$ & 71.9 & 81.9 & 60.7 & 75.0 & .147 \\
\hline \multicolumn{6}{|l|}{ Medical } \\
\hline Medical comorbidities & $0.7(0.8)$ & $1.3(1.3)$ & $0.7(1.0)$ & $0.9(0.9)$ & .003 \\
\hline Hepatitis $\mathrm{C}$ virus $(\%)$ & 14.1 & 21.7 & 14.3 & $10.8^{4}$ & .413 \\
\hline Est. duration of infection (years) & - & $14.0(8.8)^{2}$ & - & $10.3(7.7)^{4}$ & .021 \\
\hline Plasma RNA Detectable (\%) & - & $23.5^{2}$ & - & $38.5^{4}$ & .092 \\
\hline Current CD4 count $(\text { cells } / \mu \mathrm{L})^{\mathrm{a}}$ & - & $\begin{array}{c}546.5 \\
(398.8,750.8)^{2}\end{array}$ & - & $\begin{array}{c}485.0 \\
(331.0,685.0)^{4}\end{array}$ & .600 \\
\hline Nadir CD4 count $(\text { cells } / \mu \mathrm{L})^{\mathrm{a}}$ & - & $\begin{array}{c}180.0 \\
(73.0,323.0)\end{array}$ & - & $\begin{array}{c}216.5 \\
(43.0,390.3)\end{array}$ & .312 \\
\hline AIDS (\%) & - & 59.0 & - & 52.5 & .494 \\
\hline $\begin{array}{l}\text { Antiretroviral therapy }(\% \\
\text { prescribed) }\end{array}$ & - & 86.8 & - & 77.5 & .202 \\
\hline
\end{tabular}

Note. Values are means (SD) or valid population \% values. $H I V$ human immunodeficiency virus, $G D S$ Global Deficit Score, POMS Profile of Mood States, RNA Ribonucleic acid, CD4 cluster of differentiation, AIDS acquired immunodeficiency syndrome. ${ }^{\text {a }}$ Based on median and interquartile range (IQR) scores. Bold indicates $p<.05 .{ }^{1} \mathrm{n}<64,{ }^{2} \mathrm{n}<83,{ }^{3} \mathrm{n}<29,{ }^{4} \mathrm{n}<40$ 
Figure 1. Line graph depicting mean sample-based neurocognitive z-scores for Black and White participants with and without HIV. Mean (SE) global z-scores for each group were as follows: White- .20 (.07), White+ .02 (.06), Black- -.04 (.11), Black+ -.36 (.09).

$* p<.05 * * p<.001$

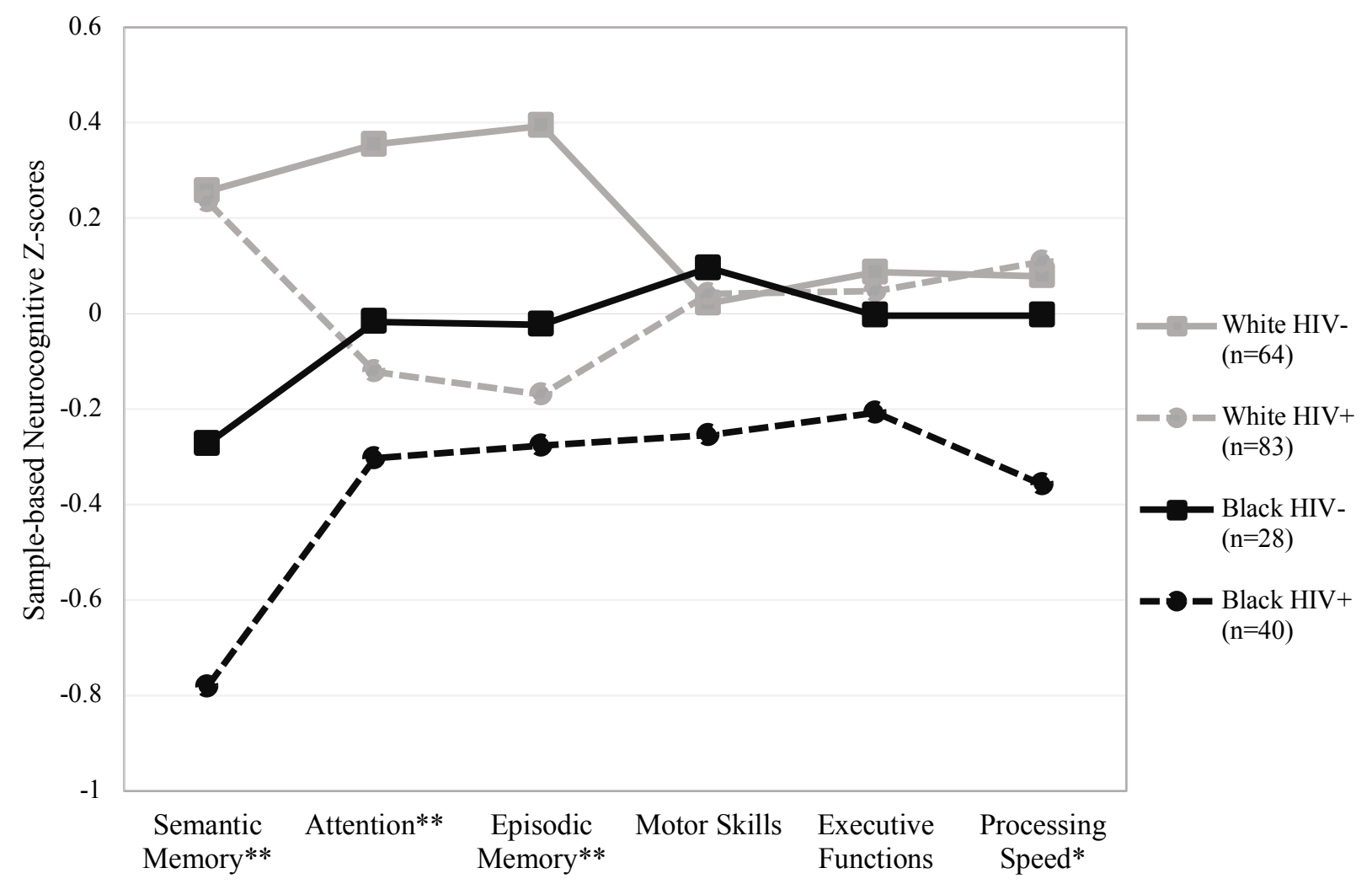

Neurocognitive Domain 
Figure 2. A stacked column chart displaying rates of mild (i.e., functionally intact) and major (i.e., functionally impaired) neurocognitive disorders across study groups according to Frascati criteria classifications.

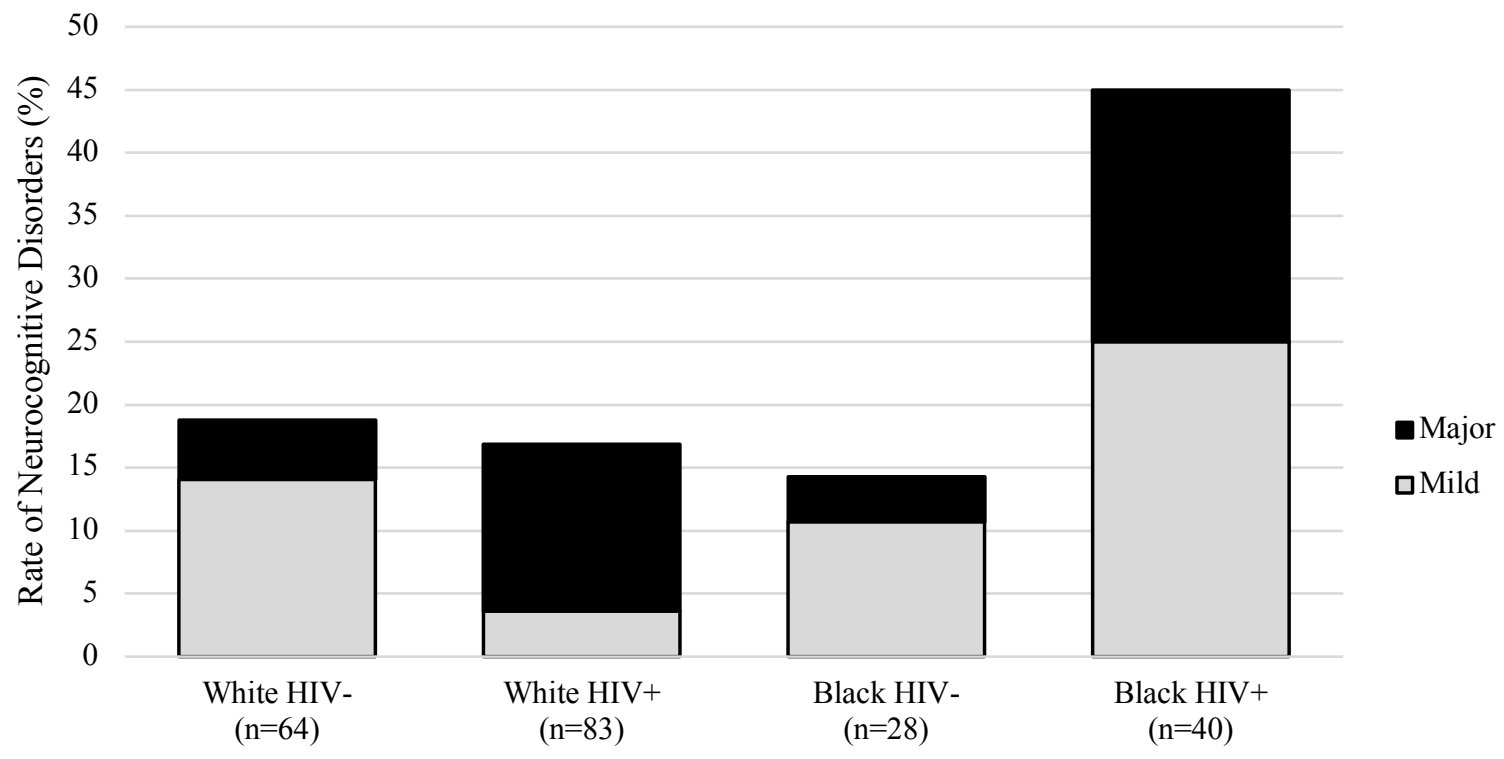


Figure 3. A scatterplot of global functional impairment and global neurocognitive performance in Black $(\mathrm{n}=40)$ and White $(\mathrm{n}=83)$ persons with HIV.

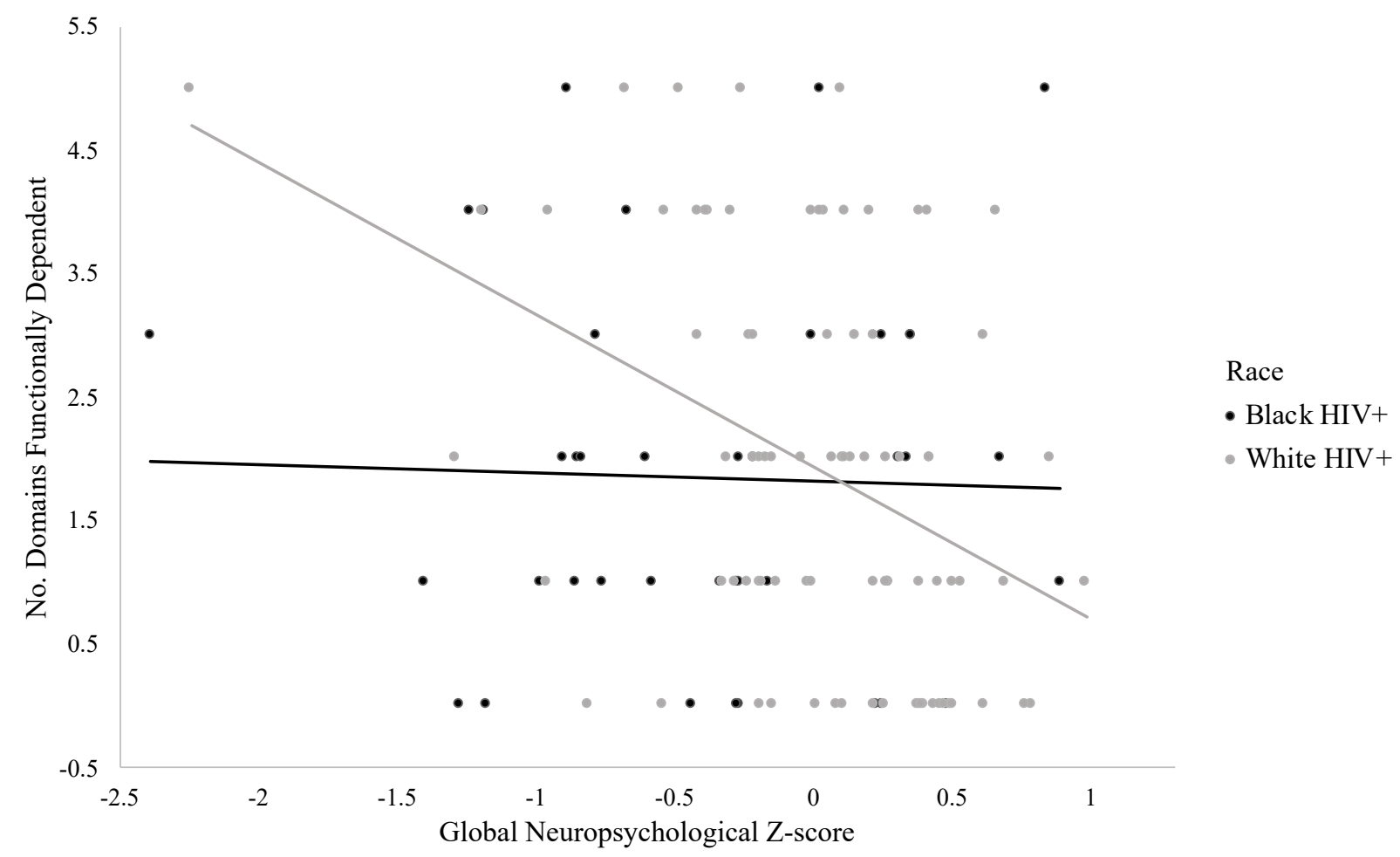

